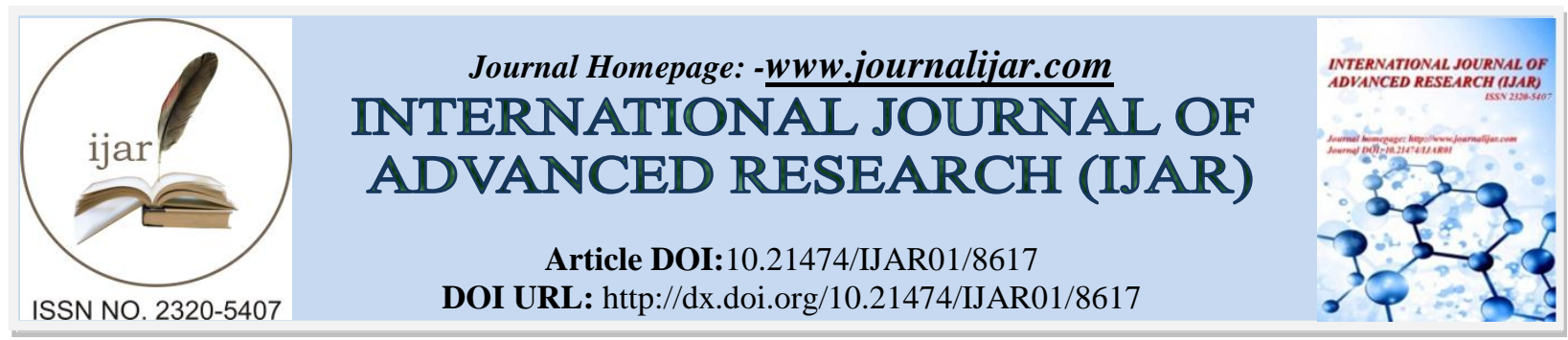

RESEARCH ARTICLE

\title{
STUDY OF THE ZIZIPHUS JOAZEIRO PEEL FOR INDIGO BLUE ADSORPTION.
}

\author{
Joselito Nardy Ribeiro ${ }^{1}$, Araceli Veronica Flores Nardy Ribeiro ${ }^{2}$, Flavio Cunha Monteiro ${ }^{1}$, Marcus Vinicius \\ VJ Licinio ${ }^{1}$ and Madson de Godoi Pereira ${ }^{3}$. \\ 1. Federal University of Espirito Santo State, Vitoria ES, Brazil. \\ 2. Federal Institute of Espirito Santo, Vila Velha ES, Brazil. \\ 3. University of Bahia State, Salvador BA, Brazil.
}

\section{Manuscript Info}

Manuscript History

Received: 05 January 2018

Final Accepted: 07 February 2019

Published: March 2019

\section{Abstract}

The Zizyphus joazeiro Mart peel (ZJP) was evaluated to remove the indigo blue (IB) in aqueous medium through the adsorption process. The high value of the maximum adsorptive capacity $\left(50 \mathrm{mg} \cdot \mathrm{g}^{-1}\right)$ of ZJP for this textile dye proves that this natural adsorbent is efficient in IB adsorption. Tests on glass columns support this claim. ZJP was more efficient (90.5\%) than activated carbon $(15.2 \%)$ in the removal of IB present in water.

Copy Right, IJAR, 2019,. All rights reserved.

\section{Introduction:-}

Dyes, including textiles dyes, are an important class of pollutants in the aquatic environment (Gupta \& Suhas, .2009). Every year a great deal of textile dyes are disposed of in the environment through industrial effluents that do not receive adequate treatment. Waste of the industrial textile dye containing indigo blue (IB) and its derivative anthranilic acid are hazardous to the aquatic environment (Zhu et al., 2016). Therefore, studies on methodologies for the removal of this and other dyes pollutants are of extreme importance. Among the most studied techniques is the adsorption using simple (Raymundo et al., 2010) or modified (Zhu et al., 2016) natural adsorbents. The use of this material is justified not only by its adsorption efficiency but also by its high availability and low cost (Gupta \& Suhas, .2009). Ribeiro et al (2018) studied the use of liquorice Glycyrrhiza glabra L. root powder for the removal of congo red dye and IB in aqueous solutions. Pereira et al (2009) evaluated the vermicompost for tinted organic cationic dyes retention. Raymundo et al (2010) utilized sugar-cane bagasse as bioadsorbent in the textile wastewater treatment contaminated with congo red dye. Munagapat et al (2018) investigated the removal of anionic dyes (Reactive Black 5 and Congo Red) from aqueous solutions using banana peel powder as an natural adsorbent. Zhu et al (2016) utilized cellulose and chitosan for removal of toxic IB. Sun et al (2018) evaluated the use of pristine and modified radix Angelicae dahuricae (Baizhi) residue for the adsorption of methylene blue from aqueous solution. Kebaili et al (2018) studied orange industry residues as an adsorbent for methylene blue. Various other low cost adsorbents have been used for the treatment of water containing dyes (Gupta \& Suhas, 2009). However, Zizyphus joazeiro Mart, a Brazilian northeastern plant, only used for medicinal and other applications not was studied for dye removal in water. In this study we investigated the use of Zizyphus joazeiro Mart. peel (ZJP) as a possible IBremoving agent in water. For this study the surface of ZJP was studied by scanning electron microscopy, besides tests on the influence of grain size and ZJP mass on the removal of IB in water. Tests were also performed under the influence of stirring time in the adsorptive process. Subsequently, the maximum adsorptive capacity (MAC) of ZJP for IB was determined. The MAC value represents the amount of IB that can be retained in $1.0 \mathrm{~g}$ of ZJP. Finally, filtration studies were performed on glass columns and the efficiency of ZJP was compared to the activated carbon for the removal of IB.

Corresponding Author:-Joselito Nardy Ribeiro.

Address:-Federal University of Espirito Santo State, Vitoria ES, Brazil. 


\section{Material and methods:- \\ Materials:-}

Indigo blue (IB) was purchased from Tupy Industry (São Paulo-SP, Brazil) for the preparation of aqueous solutions. The Zizyphus joazeiro Mart. peel (ZJP) was obtained from Empório Cupano Comercial LTDA (Pinhais-PR, Brazil). The activated carbon (AC) was obtained from Casa das Químicas (Flores da Cunha-RS, Brazil). The following equipment was used in laboratory tests: laboratory oven (Quimis Q-317 B model), particle size sieves (Granutest), Analytical balance (Shimadzu AY 220 model), sputter coater (Shimadzu, IC-50 Ion Coater model), scanning electron microscope (Shimadzu, SSX 550 model), pHmeter (PHTEK), magnetic stirrer (Warmnest), UV/Vis spectrophotometer (Even model), vacuum filtration pump (Prismatec) and microcentrifuge 13,000 rpm (Evlab).

\section{Methods:-}

\section{Preparation of the ZJP:-}

The Zizyphus joazeiro Mart. peel (ZJP) was triturated in an industrial blender to obtain particles between 2.38 and $4.29 \mathrm{~mm}, 1.19$ and $2.38 \mathrm{~mm}$ and finally particles smaller than $1.19 \mathrm{~mm}$. The particle sizes were selected in particle size sieves (Granutest). Subsequently the material was washed 10 times in distilled water (pH 7.0) and dried in a laboratory over for $35 \mathrm{hrs}$ at $40^{\circ} \mathrm{C}$. After drying, the material was stored in hermetically sealed plastic bottles.

\section{Granulometry adsorption tests:-}

This step had as purpose to evaluate which particle size would be more efficient in the dye adsorption. For this aqueous solutions $(\mathrm{pH} 7.0)$ containing IB $(1,000 \mathrm{mg} / \mathrm{L})$ were stirred $(1,000 \mathrm{rpm})$ at 5 minutes and $25^{\circ} \mathrm{C}$ in the presence of $4.0 \mathrm{~g}$ of ZPJ of different particle sizes $(2.38-4.29 \mathrm{~mm}$ or $1.19-2.38 \mathrm{~mm}$ or less than $1.19 \mathrm{~mm})$. Subsequently the solutions were filtered at vacuum for retention of the ZJP containing IB dye. The supernatants were centrifuged at 13,000 rpm for 3 minutes to remove ZJP residues. After centrifugation the supernatants were analyzed in a spectrophotometer at $573 \mathrm{~nm}$ to IB to evaluate the amount (\%) of this dye retained in the ZJP.

\section{Scanning electron microscopy (SEM) study:-}

Analysis of the surfaces of the ZJP particles $(<1.19 \mathrm{~mm})$ were performed using a scanning electron microscope. Before analysis the ZJP particles were immersed in a thin layer of gold using the spray coating. Subsequently the surface of this sample was visualized in SEM (electron beam of $20 \mathrm{kV}$ ). The use of higher voltage electron beams has been attempted. However, at $25 \mathrm{kV}$ the material was destroyed, making it impossible the visualization of the ZJP surface.

\section{Influence of ZJP mass in adsorptive process}

Aqueous solutions $(\mathrm{pH} 7.0)$ containing IB $(1,000 \mathrm{mg} / \mathrm{L})$ were stirred $(1,000 \mathrm{rpm})$ at 5 minutes and $25^{\circ} \mathrm{C}$ in the presence of different ZPJ $(<1.19 \mathrm{~mm})$ mass $(0.25,0.50,1.0,1.5,2.0,2.5,3.0,3.5,4.0$, and $5.0 \mathrm{~g})$. Subsequently the solutions were filtered at vacuum (figure 2) for retention of the adsorbent containing IB dye. The supernatants were centrifuged at 13,000 rpm for 3 minutes to remove ZJP residues. After centrifugation the supernatants were analyzed in a spectrophotometer at $573 \mathrm{~nm}$ to IB to evaluate the amount (\%) of dye retained in the ZJP. Each mass point was made in triplicate.

\section{Influence of stirring time:-}

Aqueous solutions $(\mathrm{pH} 7.0)$ containing $1,000 \mathrm{mg} / \mathrm{L} \mathrm{IB}$ were stirred $(1,000 \mathrm{rpm})$ in the presence of $1.5 \mathrm{~g} \mathrm{ZJP}(<1.19$ $\mathrm{mm})$ at $25^{\circ} \mathrm{C}$ at different times $(0.5,2.0,3.0,4.0,5.0,6.0,7.0,8.0,9.0$, and 10.0 minutes). Subsequently the solutions were filtered at vacuum (figure 2) for retention of the adsorbent containing IB dye. The supernatants were centrifuged at 13,000 rpm for 3 minutes to remove ZJP residues. After centrifugation the supernatants were analyzed in a spectrophotometer at $573 \mathrm{~nm}$ to IB to evaluate the amount (\%) of this dye retained in the ZJP. Each time point was made in triplicate

\section{Influence of dye concentration and determination of MAC value:-}

The objective of this step was to calculate the MAC value using the Langmuir Mathematical Model. This value represents the maximum amount of IB that can be retained in $1 \mathrm{~g}$ of ZJP. For this, aqueous solutions (pH 7.0) containing increasing concentrations of IB were stirred at $1,000 \mathrm{rpm}$ for 4 minutes (time previously optimized) at $25^{\circ} \mathrm{C}$ in the presence of $1.5 \mathrm{~g}$ (mass previously optimized) of ZJP. The supernatants were centrifuged at 13,000 rpm for 3 minutes to remove ZJP residues. After centrifugation the supernatants were analyzed in a spectrophotometer at $573 \mathrm{~nm}$ to IB to evaluate the amount (\%) of dye retained in the ZJP. 


\section{Experiments in glass columns:-}

Three glass columns $(38 \times 3 \mathrm{~cm})$ were filled with $8.2 \mathrm{~g}$ of activated carbon (AC) in $5.0 \mathrm{~cm}$ of height and $20 \mathrm{~g}$ of gravel in $9.0 \mathrm{~cm}$ of height. Another three glass columns were equally filled, but with the addition of $10.0 \mathrm{~g}$ of ZJP in $5.0 \mathrm{~cm}$ of height. Subsequently $50 \mathrm{ml}$ of $1000 \mathrm{mg} / \mathrm{L} \mathrm{IB}$ aqueous solutions were percolated through the columns. The flow rate used was $2.0 \mathrm{ml} / \mathrm{min}$. for all columns (optimized in our laboratory). The filtered solutions were centrifuged at $13,000 \mathrm{rpm}$ and analyzed in a spectrophotometer at $573 \mathrm{~nm}$ to IB to evaluate the amount (\%) of this dye retained in the column.

\section{Results and discussion:-}

Granulometry adsorption tests:-

The results of this analysis demonstrated that the smaller particles $(<1.19 \mathrm{~mm})$ provided higher adsorption for IB (figure 1). After these tests, the particles of smaller size $(<1.19 \mathrm{~mm})$ were selected for analysis by scanning electron microscopy (SEM) and other later stages. These results of granulometry can be explained by the increase of the contact surface provided by smaller particles. However, this is not always true. For example, Raymundo et al. (2010) demonstrated that larger sugarcane bagasse particles were more efficient for removing congo red dye than smaller particles.

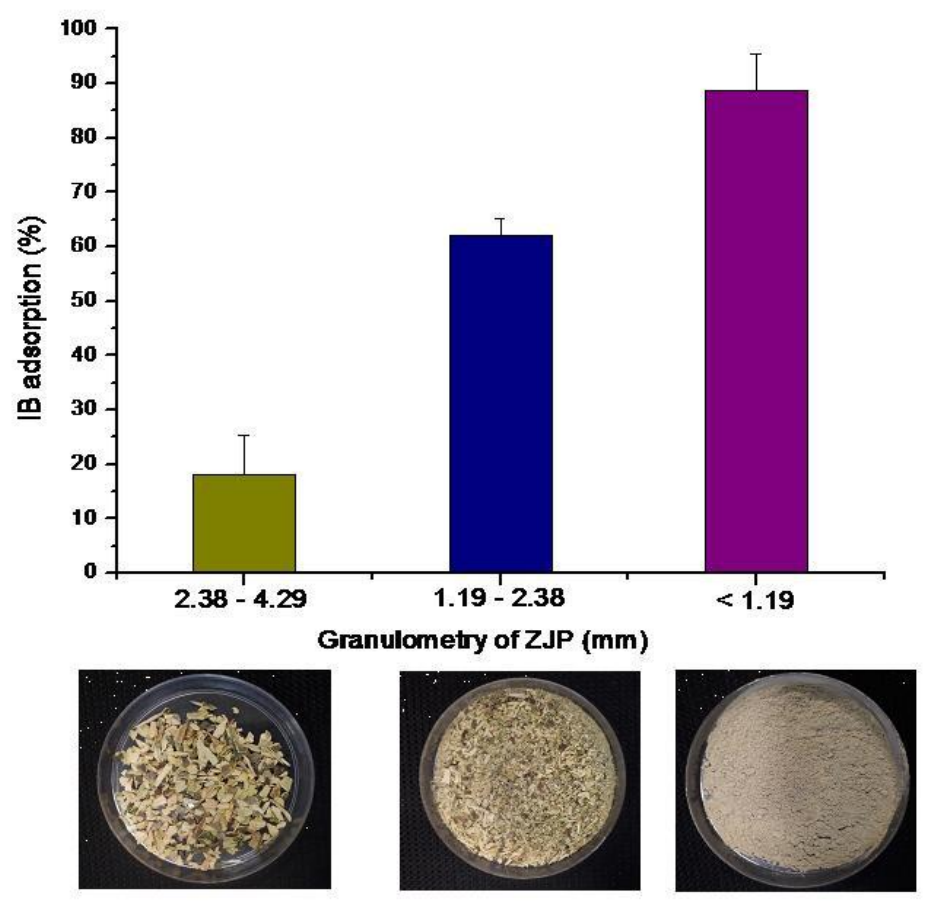

Figure 1:- Influence of granulometry of ZJP particles in the IB adsorption (\%).

\section{Scanning electron microscopy (SEM) study:-}

The visualization of the ZJP particles on a larger scale (20.0 micrometers) appears to reveal a heterogeneous surface (figure 2). However, the smaller scale visualization (2.0 micrometers) showed that the surface is homogeneous without irregularities and arranged in the form of smooth plates (figure 2). This is not an interesting feature for the adsorption process, since the irregular surfaces are indicated for an efficient adsorption (Ribeiro et al., 2016). The morphological homogeneous characteristics of ZJP surface suggest relative disadvantage in this physical interaction between this adsorbent and IB. However, it was observed that some adsorbents which have homogeneous surface are also capable of interacting with pollutants in an aqueous medium. These interactions occur due to the presence of chemical groups of the adsorbents and not only to the physical deposition of the pollutants molecules on heterogeneous surface (Ribeiro et al., 2011). 
(A)

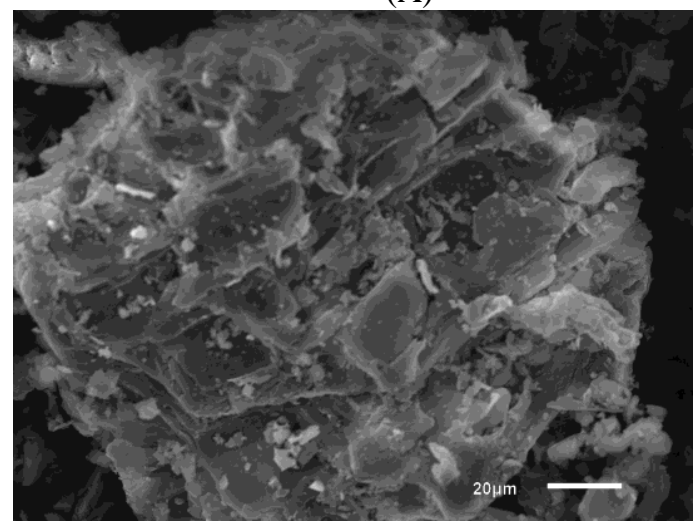

(B)

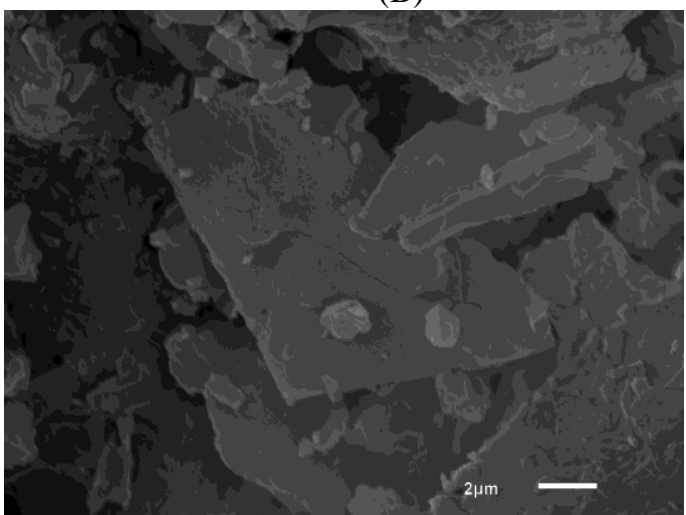

Figure 2:- Scanning electron microscopy of the ZJP particles on a larger scale (20.0 micrometers) (A) and smaller scale (2.0 micrometers) (B)

Influence of ZJP mass in adsorptive process:-

Pereira et al (2009) demonstrated that the increase in the mass of the vermicompost adsorbent causes an increase in the adsorption percentage of the violet crystal dye up to $5 \mathrm{~g}$ remaining constant from that point. Ribeiro et al (2018) demonstrated that the increase in the mass of liquorice Glycyrrhiza glabra L. root powder increased the percentage of Congo red and IB adsorption. However, from a given mass the percentage of adsorption remained constant reaching an equilibrium adsorption condition. A similar result was found in this study. The increase in ZJP mass resulted in an increase in the IB adsorption percentage. However, considering the standard deviations, from $1.5 \mathrm{~g}$ the adsorption remained constant at around 84\% (figure 3). The mass selected for the other stages of this study was 1.5 g.

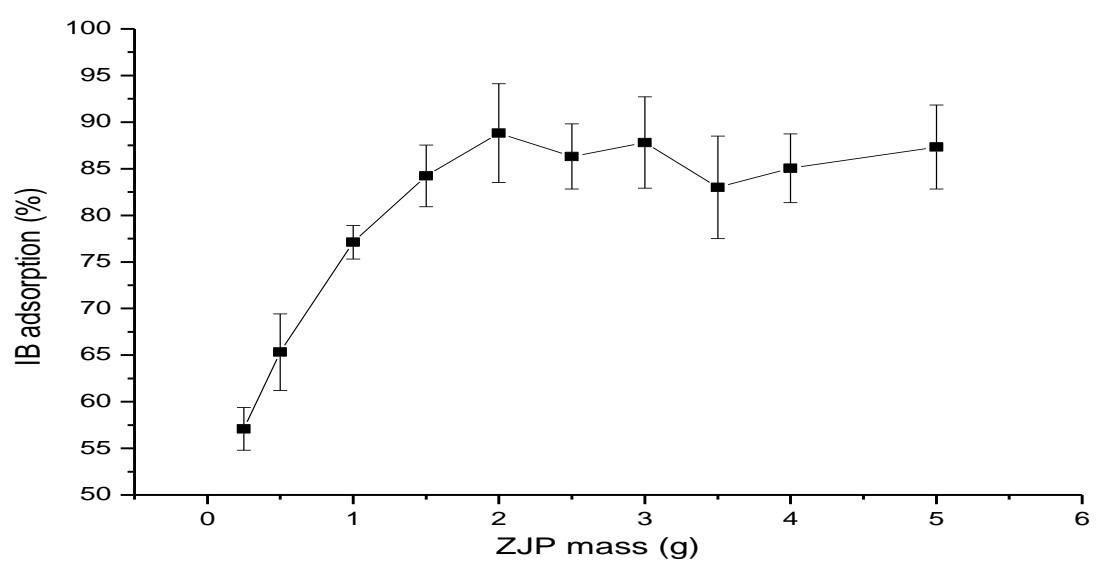

Figure 3:- Influence of ZJP mass (g) in the IB adsorption (\%)

\section{Influence of stirring time:-}

The data obtained in this experiment revealed that the increase in stirring time causes increase in adsorption up to a range of 4 to 7 minutes. Already from 8 to 10 minutes a decrease in the percentage of adsorption of IB by ZJP occurs (figure 4). This observation indicates that occurred adsorption followed by desorption. Raymundo et al (2010) and Ribeiro et al (2016) also observed similar behavior when studying the adsorption of organic pollutants by natural adsorbents. The time of 4 minutes was selected for the next step of this work. 


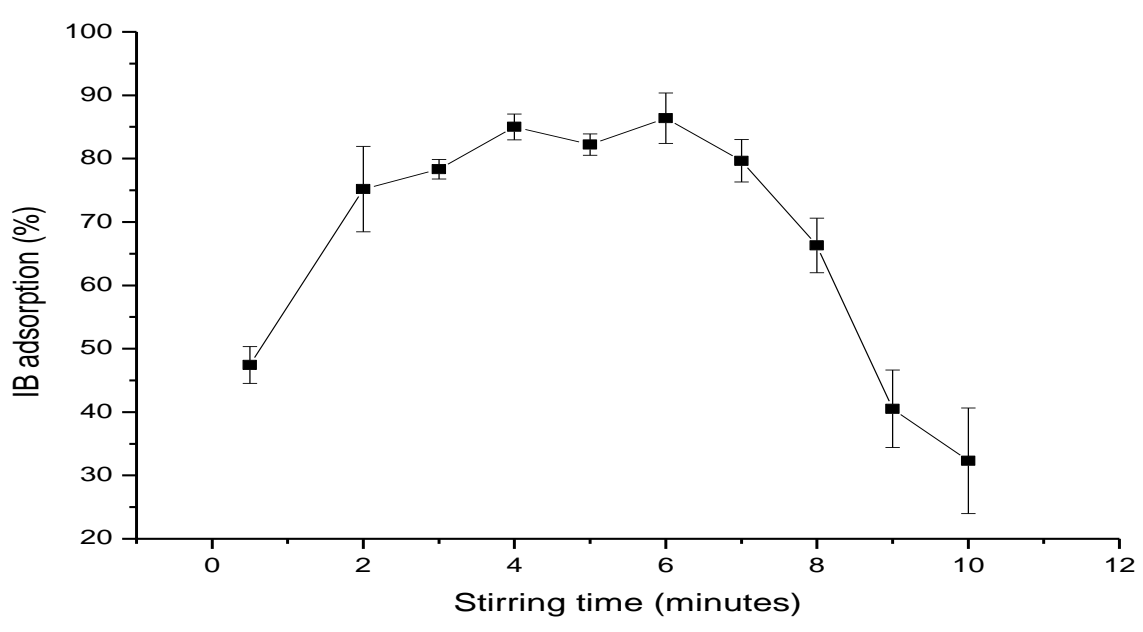

Figure 4:- Influence of stirring time in the IB adsorption (\%)

Influence of dye concentration and determination of MAC value:-

It was expected that the results obtained in this step would allow the calculation of the MAC value of ZJP for IB as described in Ribeiro et al (2018). The equations that would be used are described below (Langmuir Mathematical Model):

equations 1 and 2 allow the calculation of the MAC value

Equation (1)

$$
\begin{aligned}
& q=\left(\frac{a \cdot b \cdot \text { Ceq }}{(1+a \cdot \text { Ceq })}\right) \\
& \text { Equation (2) }(y=a+b x) \\
& \frac{C e q}{q}=\left(\frac{1}{(a \cdot b)}+\frac{1}{b} \cdot \text { Ceq }\right)
\end{aligned}
$$

Where $q$ is the quantity of adsorbate adsorbed in the adsorbent $(\mathrm{mg} / \mathrm{g}), a$ is a constant related to adsorption energy $(\mathrm{L} / \mathrm{mg}), b$ is the maximum adsorbate adsorption capacity of the adsorbent $(\mathrm{mg} / \mathrm{g})$, and $\mathrm{Ceq}$ is the equilibrium concentration of adsorbate in supernatant $(\mathrm{mg} / \mathrm{L})$. Finally, the equation 2 can be, under favorable conditions, obtained through the linearization of equation 1 . The equation 2 permits exact calculations of $b$ values. From this equation, it is possible to calculate the maximum adsorption capacity $\left(b^{-1}=\mathrm{MAC}\right)$ of adsorbent to adsorbate. The favorable conditions for the adsorption of IB by ZJP allowed to obtain a favorable isotherm (figure 5) that, after linearization (figure 6), allowed the calculation of the MAC value $\left(50.0 \mathrm{mg} \cdot \mathrm{g}^{-1}\right)$. This value is considerably higher than that obtained by Ribeiro et al (2018) (1.696 mg.g $\mathrm{g}^{-1}$ ) who studied the adsorption of IB by liquorice Glycyrrhiza glabra L. root powder. Moreover, it is a very high value when compared to values obtained by other authors employing other dyes and adsorbents. For example, Namasivayam \& Arasi (1997) found a MAC value of $4.05 \mathrm{mg} . \mathrm{g}^{-}$ ${ }^{1}$ of red mud adsorbent to Congo red dye. Raymundo et al. (2010) obtained a MAC value of $4.43 \mathrm{mg} \cdot \mathrm{g}^{-1}$ of sugarcane bagasse adsorbent to Congo red dye and Maghri et al. (2012) found a MAC value of $3.43 \mathrm{mg} \cdot \mathrm{g}^{-1}$ of Mytilus edulis shells to methylene blue dye. 


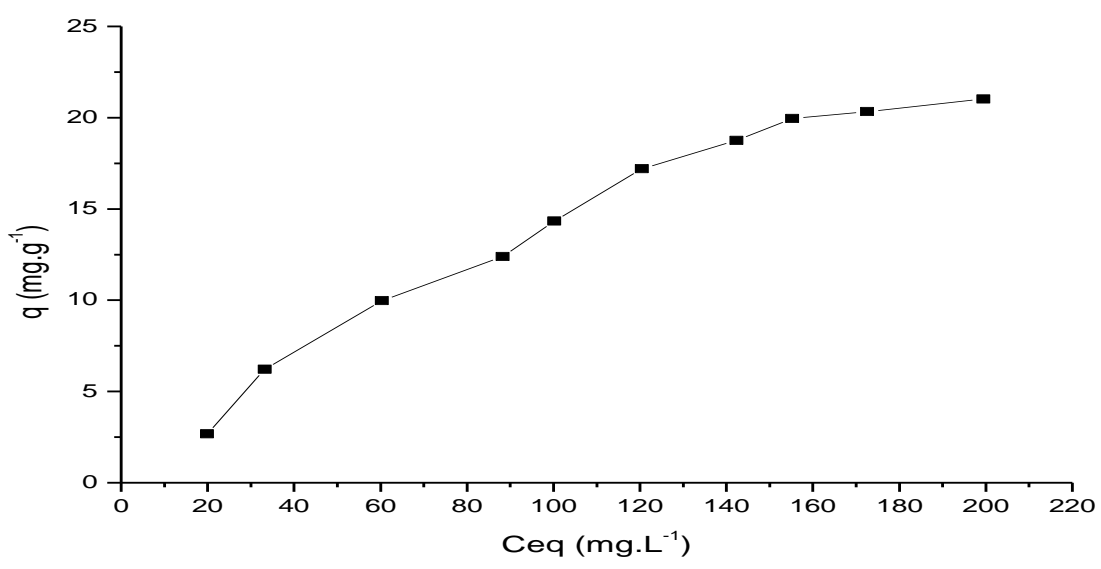

Figure 5:- Isotherm of IB adsorption by ZJP

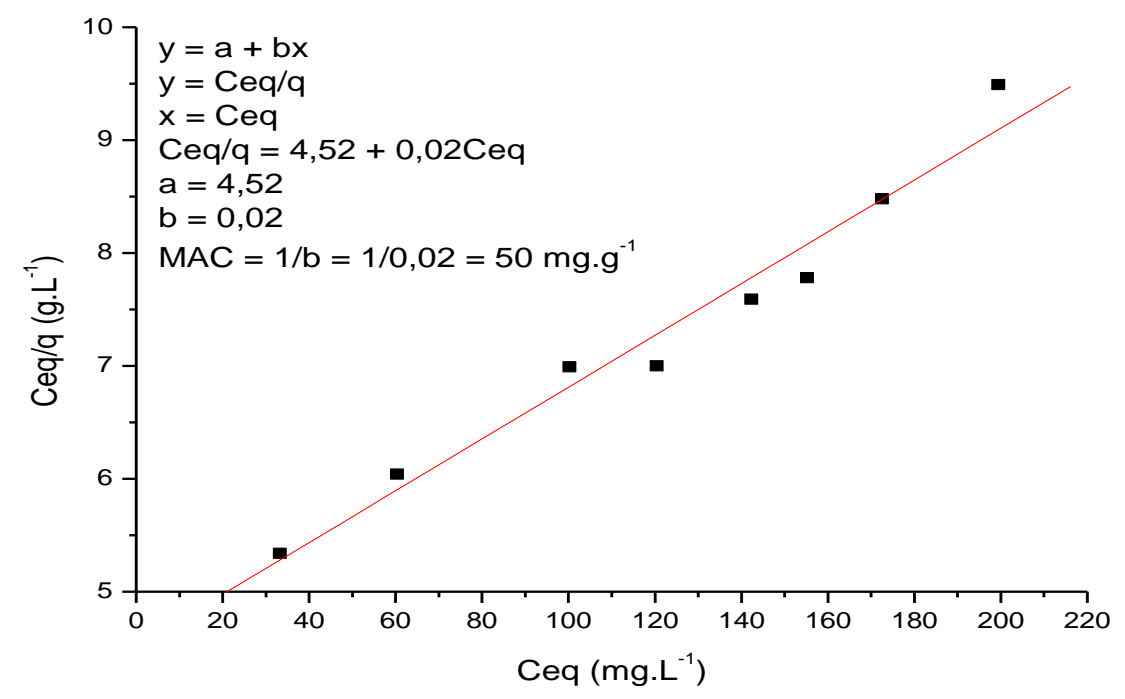

Figure 6:- Linearized isotherm of IB adsorption by ZJP for MAC calculation

\section{Experiments in glass columns:-}

The results using filtration in glass columns revealed that the retention of dye in the columns is greater in the presence than in the absence of ZJP. Columns containing only gravel and activated carbon were able to remove 15.2 $\%$ of IB. However, the columns containing gravel, activated carbon and ZJP removed $90.5 \%$ of dye (figure 7). Figure 8 shows the water bleaching after passage through the column and the retention of the dye on the surface of the ZJP layer on the column. Ribeiro et al (2018) obtained 98.2\% of IB retention using Glycyrrhiza glabra L. root powder in glass column, which can be considered an excellent result. 


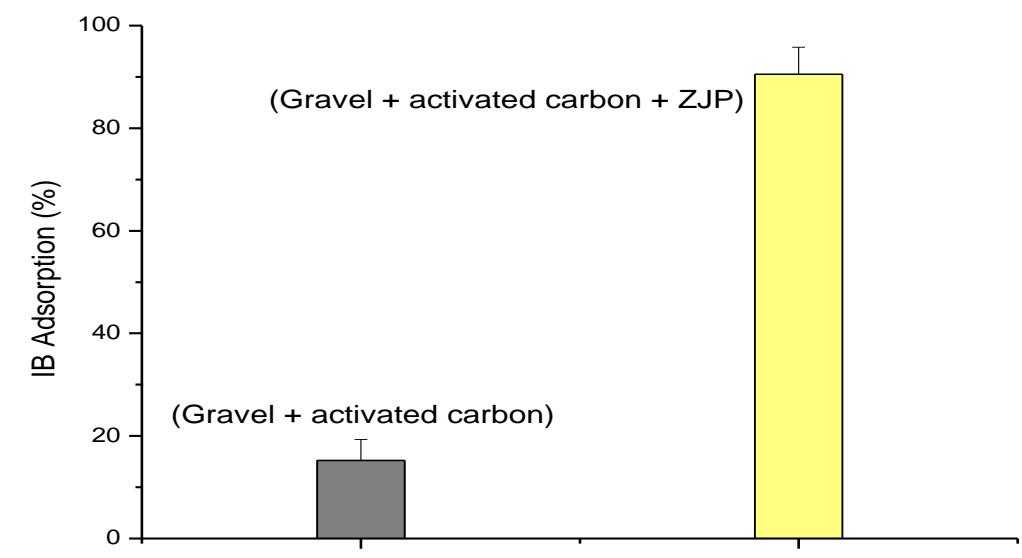

Figure 7:- Percentage of IB adsorption in glass columns in the presence and absence of ZJP
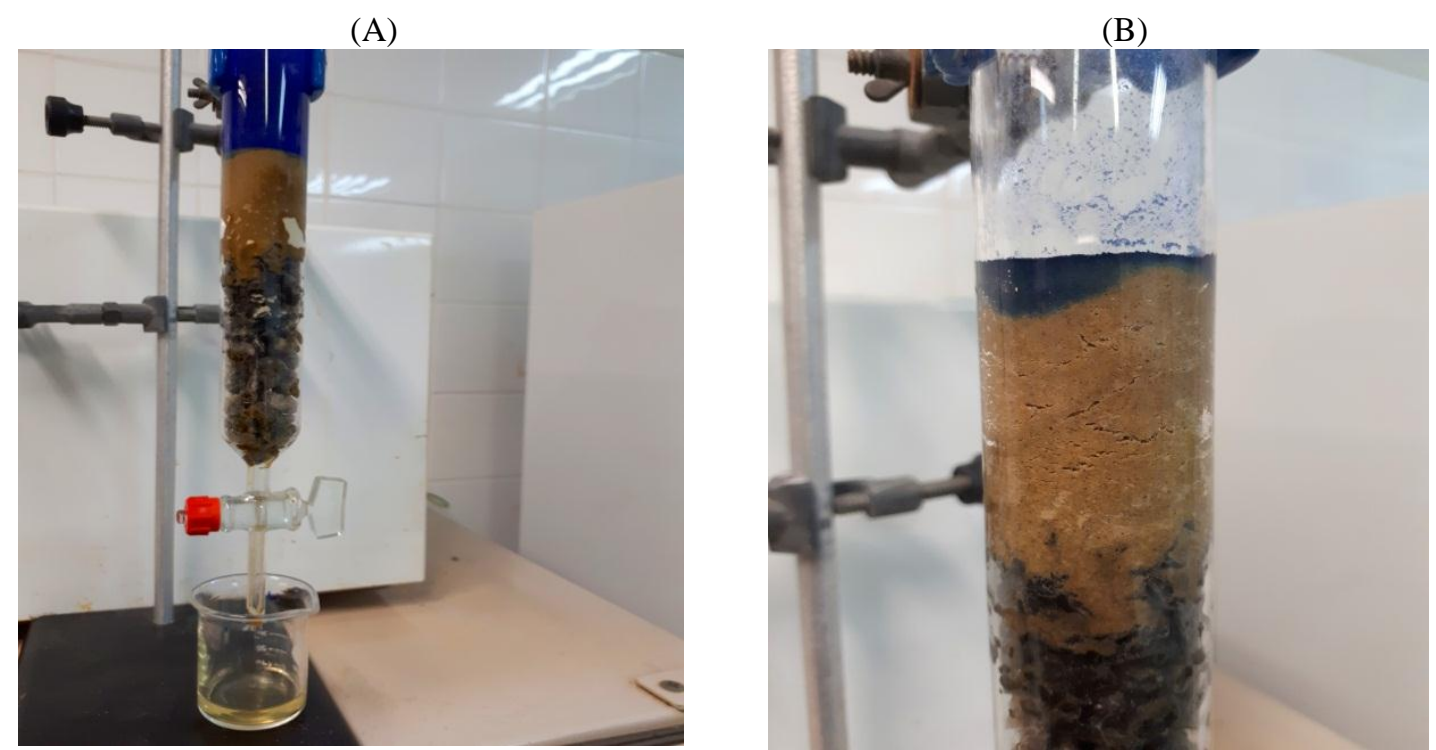

Figure 8:- Water bleaching after passage through the column (A) and the retention of the dye (B) on the surface of the ZJP layer on the column.

\section{Conclusion:-}

The results of the experiments show that ZJP may possibly be used as a natural adsorbent alternative in the treatment of textile effluents containing IB. The high MAC value and the high adsorption percentage of IB in columns support this assertion.

\section{Acknowlegdments:-}

The authors would like to thank the Federal Institute of Espírito Santo (Vitória, ES, Brazil) by financial support and Laboratory of Cellular Ultra structure Carlos Alberto Redins of Federal University of Espírito Santo (Vitória, ES, Brazil) for the use of scanning electron microscope. 


\section{References:-}

1. Gupta, V. K., Suhas. Application of low-cost adsorbents for dye removal - A review. Journal of Environmental Management, 90, 2313-2342, 2009.

2. Kebaili, M., Djellali, S., Radjai, M., Drouiche, N., Lounici, H. Valorization of orange industry residues to form a natural coagulant and adsorbent. Journal of Industrial and Engineering Chemistry, 64, 292-299, 2018.

3. Maghri, I., Amegrissi, F., Mohamed, E., Kenz, A., Tanane, O., Mohamed, T., Salouhi, M. Comparison of adsorption of dye onto low-cost adsorbents. Global Journal of Science Frontier Research Chemistry, 12, 4, 1-6, 2012

4. Munagapat, V.S., Yarramuthi, V., Kim, Y., Lee, K.M., Kim, D.S. Removal of anionic dyes (Reactive Black 5 and Congo Red) from aqueous solutions using Banana Peel Powder as an adsorbent. Ecotoxicology and Environmental Safety, 148, 601-607, 2018.

5. Namasivayam, C., Arasi, J.S.E. Removal of congo red from wastewater by adsorption onto waste red mud. Chemosphere, 34, 401-417, 1997

6. Pereira, M.G., Korn, M., Santos, B.B., Ramos, M.G. Vermicompost for tinted organic cationic dyes retention. Water Air Soil Pollution, 200, 227-235, 2009

7. Raymundo, A.S., Zanarotto, R., Belisário, Marciela., Pereira, M.G., Ribeiro, J.N., Ribeiro, A.V.F.N. Evaluation of sugar-cane bagasse as bioadsorbent in the textile waste water treatment contaminated with carcinogenic congo red dye. Brazilian Archives of Biology and Technology, 53, 931-938, 2010.

8. Ribeiro, A. V. F. N., Belisário, M., Galazzi, R. M., Balthazar, D. C., Pereira, M. G., Ribeiro, J. N. Evaluation of two bioadsorbents for removing paracetamol from aqueous media. Electronic Journal of Biotechnology, 14, 6, 1-10, 2011.

9. Ribeiro, A.V.F.N., Silva, A.R., Cunha, T.P., Santos, R.T.L., Oliveira, J.P., Pereira, E.V., Licinio, M.V.V.J., Pereira, M.G., Santos, A.V., Ribeiro, J.N. Banana peel for acetylsalicylic acid retention. Journal of Environmental Protection, 7, 1850-1859, 2016

10. Ribeiro, A.V.F.N., Silva, A. R., Silvares, P. H. S., Loiola, A. O., Monteiro, F. C., Pereira, M. G., Ribeiro, J. N. Evaluation of liquorice Glycyrrhiza glabra L. root powder as a new adsorbent in the removal of textile dyes in aqueous medium. International Journal Advanced Research, 6, 8, 278-290, 2018.

11. Sun, Q., Saratale, R. G., Saratale, G. D. Kim, Dong-Su. Pristine and modified radix Angelicae dahuricae (Baizhi) residue for the adsorption of methylene blue from aqueous solution: A comparative study. Journal of Molecular Liquids, 265, 36-45, 2018.

12. Zhu, X., Bao, L., Wei, Y., Ma, J., Kong, Y. Removal of toxic indigo blue with integrated biomaterials of sodiumcarboxymethyl cellulose and chitosan. International Journal of Biological Macromolecules, 91, 409-415, 2016. 